\title{
QUASI-MORPHISMS ON THE GROUP OF AREA-PRESERVING DIFFEOMORPHISMS OF THE 2-DISK VIA BRAID GROUPS
}

\author{
TOMOHIKO ISHIDA
}

(Communicated by Michael Wolf)

\begin{abstract}
Recently Gambaudo and Ghys proved that there exist infinitely many quasi-morphisms on the group $\operatorname{Diff}_{\Omega}^{\infty}\left(D^{2}, \partial D^{2}\right)$ of area-preserving diffeomorphisms of the 2-disk $D^{2}$. For the proof, they constructed a homomorphism from the space of quasi-morphisms on the braid group to the space of quasimorphisms on $\operatorname{Diff}_{\Omega}^{\infty}\left(D^{2}, \partial D^{2}\right)$. In this paper, we study this homomorphism and prove its injectivity.
\end{abstract}

\section{INTRODUCTION}

For a group $G$, a function $\phi: G \rightarrow \mathbb{R}$ is called a quasi-morphism if the real valued function on $G \times G$ defined by

$$
(g, h) \mapsto \phi(g h)-\phi(g)-\phi(h)
$$

is bounded. The real number

$$
D(\phi)=\sup _{g, h \in G}|\phi(g h)-\phi(g)-\phi(h)|
$$

is called the defect of $\phi$. We denote the $\mathbb{R}$-vector space of quasi-morphisms on the group $G$ by $\hat{Q}(G)$. By definition, bounded functions on groups are quasi-morphisms. Hence we denote the set of bounded functions on the group $G$ by $C_{b}^{1}(G ; \mathbb{R})$ and consider the quotient space $Q(G)=\hat{Q}(G) / C_{b}^{1}(G ; \mathbb{R})$. A quasi-morphism $\phi: G \rightarrow \mathbb{R}$ is said to be homogeneous if the equation

$$
\phi\left(g^{p}\right)=p \phi(g)
$$

holds for any $g \in G$ and $p \in \mathbb{Z}$. For any quasi-morphism $\phi$, a homogeneous quasimorphism $\tilde{\phi}$ is defined by setting

$$
\tilde{\phi}(g)=\lim _{p \rightarrow \infty} \frac{1}{p} \phi\left(g^{p}\right) .
$$

The limit always exists for each element $g$ of $G$. The new function $\tilde{\phi}$ is in fact a quasimorphism equal to the original quasi-morphism $\phi$ as an element of $Q(G)$. Thus we can identify the vector space of homogeneous quasi-morphisms on the group $G$ with $Q(G)$. Homogeneous quasi-morphisms are invariant under conjugation. Therefore we are interested in $Q(G)$ rather than $\hat{Q}(G)$.

Received by the editors July 19, 2012 and, in revised form, September 22, 2012; October 15, 2012; October 19, 2012; December 31, 2012; and February 27, 2013.

2010 Mathematics Subject Classification. Primary 37C15; Secondary 37E30.

Key words and phrases. Area-preserving diffeomorphisms, symplectomorphisms, quasimorphisms, pseudo-characters. 
Let Diff $\Omega^{\infty}\left(D^{2}, \partial D^{2}\right)$ be the group of area-preserving $C^{\infty}$-diffeomorphisms of the 2-disk $D^{2}$, which are the identity on a neighborhood of the boundary. On the vector space $Q\left(\operatorname{Diff}_{\Omega}^{\infty}\left(D^{2}, \partial D^{2}\right)\right)$, the following theorem is known.

Theorem 1.1 (Entov-Polterovich [3], Gambaudo-Ghys [5]). The vector space $Q\left(\operatorname{Diff}_{\Omega}^{\infty}\left(D^{2}, \partial D^{2}\right)\right)$ is infinite dimensional.

To prove Theorem 1.1. Entov and Polterovich explicitly constructed uncountably many quasi-morphisms on $\operatorname{Diff}_{\Omega}^{\infty}\left(D^{2}, \partial D^{2}\right)$, which are linearly independent. After that Gambaudo and Ghys constructed countably many quasi-morphisms on $\operatorname{Diff}_{\Omega}^{\infty}\left(D^{2}, \partial D^{2}\right)$ using a different idea, which is to consider the suspension of areapreserving diffeomorphisms of the disk and average the value of the signature of the braids appearing in the suspension. By generalizing their strategy Brandenbursky [1] defined the homomorphism

$$
\Gamma_{n}: Q\left(P_{n}\left(D^{2}\right)\right) \rightarrow Q\left(\operatorname{Diff}_{\Omega}^{\infty}\left(D^{2}, \partial D^{2}\right)\right),
$$

which we review in Section 2. Here, $P_{n}\left(D^{2}\right)$ denotes the pure braid group on $n$-strands.

Let $B_{n}\left(D^{2}\right)$ be the braid group on $n$-strands. The natural inclusion $i: P_{n}\left(D^{2}\right) \rightarrow$ $B_{n}\left(D^{2}\right)$ induces the homomorphism $Q(i): Q\left(B_{n}\left(D^{2}\right)\right) \rightarrow Q\left(P_{n}\left(D^{2}\right)\right)$. In this paper, we study the homomorphism $\Gamma_{n}$ and prove the following theorem.

Theorem 1.2. The composition

$$
\Gamma_{n} \circ Q(i): Q\left(B_{n}\left(D^{2}\right)\right) \rightarrow Q\left(\operatorname{Diff}_{\Omega}^{\infty}\left(D^{2}, \partial D^{2}\right)\right)
$$

is injective.

\section{Gambaudo and Ghys' Construction AND PROOF OF THE MAIN THEOREM}

In this section, we review Gambaudo and Ghys' construction [5] of quasi-morphisms on the group Diff $\operatorname{Di}^{\infty}\left(D^{2}, \partial D^{2}\right)$ in a generalized form and prove Theorem 1.2 .

Let $X_{n}\left(D^{2}\right)$ be the configuration space of ordered $n$-tuples in the 2-disk $D^{2}$ and $x^{0}=\left(x_{1}^{0}, \ldots, x_{n}^{0}\right)$ its base point. For any $g \in \operatorname{Diff}_{\Omega}^{\infty}\left(D^{2}, \partial D^{2}\right)$ and for almost all $x=\left(x_{1}, \ldots, x_{n}\right) \in X_{n}\left(D^{2}\right)$, we define the pure braid $\gamma(g ; x)$ as the following. First we set the loop $l(g ; x):[0,1] \rightarrow X_{n}\left(D^{2}\right)$ by

$$
l(g ; x)(t)= \begin{cases}\left\{(1-3 t) x_{i}^{0}+3 t x_{i}\right\} & \left(0 \leq t \leq \frac{1}{3}\right), \\ \left\{g_{3 t-1}\left(x_{i}\right)\right\} & \left(\frac{1}{3} \leq t \leq \frac{2}{3}\right), \\ \left\{(3-3 t) g\left(x_{i}\right)+(3 t-2) x_{i}^{0}\right\} & \left(\frac{2}{3} \leq t \leq 1\right),\end{cases}
$$

where $\left\{g_{t}\right\}_{t \in[0,1]}$ is a Hamiltonian isotopy such that $g_{0}$ is the identity and $g_{1}=g$. We define the pure braid $\gamma(g ; x)$ to be the braid represented by the loop $l(g ; x)$. For almost every $x$, the braid $\gamma(g ; x)$ is well-defined. Furthermore, the braid $\gamma(g ; x)$ is independent of the choice of the flow $\left\{g_{t}\right\}$. This is because of the fact that the group $\operatorname{Diff}_{\Omega}^{\infty}\left(D^{2}, \partial D^{2}\right)$ is contractible, which is easily proved from the contractibility of the diffeomorphism group $\operatorname{Diff}^{\infty}\left(D^{2}, \partial D^{2}\right)$ of $D^{2}$ [8] and the homotopy equivalence between $\operatorname{Diff}^{\infty}\left(D^{2}, \partial D^{2}\right)$ and $\operatorname{Diff}_{\Omega}^{\infty}\left(D^{2}, \partial D^{2}\right)$ [7]. For a 
quasi-morphism $\phi$ on the pure braid group $P_{n}\left(D^{2}\right)$ on $n$-strands, we define the function $\hat{\Gamma}_{n}(\phi): \operatorname{Diff}_{\Omega}^{\infty}\left(D^{2}, \partial D^{2}\right) \rightarrow \mathbb{R}$ by

$$
\hat{\Gamma}_{n}(\phi)(g)=\int_{x \in X_{n}\left(D^{2}\right)} \phi(\gamma(g ; x)) d x .
$$

For any $\phi \in Q\left(P_{n}\left(D^{2}\right)\right)$ and $g \in \operatorname{Diff}_{\Omega}^{\infty}\left(D^{2}, \partial D^{2}\right)$ the function $\phi(\gamma(g ; \cdot))$ is integrable and thus the map $\hat{\Gamma}_{n}: \hat{Q}\left(P_{n}\left(D^{2}\right)\right) \rightarrow \hat{Q}\left(\operatorname{Diff}_{\Omega}^{\infty}\left(D^{2}, \partial D^{2}\right)\right)$ is well-defined [2]. The obtained function $\hat{\Gamma}_{n}(\phi): \operatorname{Diff}_{\Omega}^{\infty}\left(D^{2}, \partial D^{2}\right) \rightarrow \mathbb{R}$ is also a quasi-morphism, and the map $\hat{\Gamma}_{n}: \hat{Q}\left(P_{n}\left(D^{2}\right)\right) \rightarrow \hat{Q}\left(\operatorname{Diff}_{\Omega}^{\infty}\left(D^{2}, \partial D^{2}\right)\right)$ is clearly $\mathbb{R}$-linear. Moreover, it is easily checked that any bounded function on $P_{n}\left(D^{2}\right)$ is mapped to a bounded function on $\operatorname{Diff}_{\Omega}^{\infty}\left(D^{2}, \partial D^{2}\right)$, and thus the homomorphism

$$
\hat{\Gamma}_{n}: \hat{Q}\left(P_{n}\left(D^{2}\right)\right) \rightarrow \hat{Q}\left(\operatorname{Diff}_{\Omega}^{\infty}\left(D^{2}, \partial D^{2}\right)\right)
$$

induces the homomorphism $\Gamma_{n}: Q\left(P_{n}\left(D^{2}\right)\right) \rightarrow Q\left(\operatorname{Diff}_{\Omega}^{\infty}\left(D^{2}, \partial D^{2}\right)\right)$.

Remark 2.1. We see that the homomorphism $\Gamma_{n}: Q\left(P_{n}\left(D^{2}\right)\right) \rightarrow Q\left(\operatorname{Diff}_{\Omega}^{\infty}\left(D^{2}, \partial D^{2}\right)\right)$ maps the classical linking number homomorphism $\mathrm{lk}_{n}: B_{n}\left(D^{2}\right) \rightarrow \mathbb{R}$ on the braid group to a homomorphism on $\operatorname{Diff}_{\Omega}^{\infty}\left(D^{2}, \partial D^{2}\right)$. In fact, the image of lk: $B_{n}\left(D^{2}\right) \rightarrow$ $\mathbb{R}$ by the homomorphism $\Gamma_{n}\left(\mathrm{lk}_{n}\right)$ coincides with a constant multiple of the classical Calabi homomorphism on $\operatorname{Diff}_{\Omega}^{\infty}\left(D^{2}, \partial D^{2}\right)$ [4, and in this sense quasi-morphisms obtained in this way can be considered as generalizations of the Calabi homomorphism. By an argument of Brandenbursky which verifies that the homomorphism $\Gamma: \hat{Q}\left(P_{n}\right) \rightarrow \hat{Q}\left(\operatorname{Diff}_{\Omega}^{\infty}\left(D^{2}, \partial D^{2}\right)\right)$ is well-defined, it is observed that quasimorphisms obtained by the homomorphism $\hat{\Gamma}_{n}: \hat{Q}\left(P_{n}\left(D^{2}\right)\right) \rightarrow \hat{Q}\left(\operatorname{Diff}_{\Omega}^{\infty}\left(D^{2}, \partial D^{2}\right)\right)$ can be defined on the group of area-preserving $C^{1}$-diffeomorphisms of $D^{2}$, as well as the Calabi homomorphism.

Now we are ready to prove Theorem 1.2 .

Proof of Theorem 1.2. Let us suppose that a homogeneous quasi-morphism $\phi \in$ $\hat{Q}\left(B_{n}\left(D^{2}\right)\right)$ is non-trivial. Then there exists a braid $\beta \in B_{n}\left(D^{2}\right)$ such that $\phi(\beta) \neq 0$. We may assume that $\beta$ is pure. It is sufficient to prove that the homogeneous quasimorphism $\hat{\Gamma}_{n}(\phi) \in \hat{Q}\left(\operatorname{Diff}_{\Omega}^{\infty}\left(D^{2}, \partial D^{2}\right)\right)$ is also non-trivial. That is, there exists an area-preserving diffeomorphism $g \in \operatorname{Diff}_{\Omega}^{\infty}\left(D^{2}, \partial D^{2}\right)$ such that

$$
\lim _{p \rightarrow \infty} \frac{1}{p} \Gamma_{n}(\phi)\left(g^{p}\right) \neq 0 \text {. }
$$

Let $A_{i, j}$ be the pure braid which twists only the $i$-th and the $j$-th strands for $1 \leq i<j \leq n$ (see Figure 1). Since the braid $\beta$ is pure, it can be written as a composition of $A_{i, j}$ 's and their inverses. We take $n$ disjoint subsets $U_{i}$ of $D^{2}$. Furthermore, for a pair of $(i, j)$, we take subsets $V_{i, j}$ and $W_{i, j}$ of $D^{2}$ such that $U_{i} \cup U_{j} \subset W_{i, j} \subset V_{i, j}, U_{k} \cap V_{i, j}=\emptyset$ if $k \neq i, j$ and $V_{i, j}, W_{i, j}$ are diffeomorphic to $D^{2}$. Let $\left\{h_{t}\right\}_{t \in[0,1]}$ be a path in $\operatorname{Diff}_{\Omega}^{\infty}\left(D^{2}, \partial D^{2}\right)$ such that the support of $h_{t}$ is contained in the interior of $V_{i, j}$ and rotates $W_{i, j}$ once. Taking paths $\left\{h_{t}\right\}$ constructed above for all the $A_{i, j}$ 's which present $\beta$ and composing them, we have a path $\left\{g_{t}\right\}_{t \in[0,1]}$ in Diff $\operatorname{Di}^{\infty}\left(D^{2}, \partial D^{2}\right)$ with $g_{0}=i d$ which twists the $U_{i}$ 's in the form of the pure 


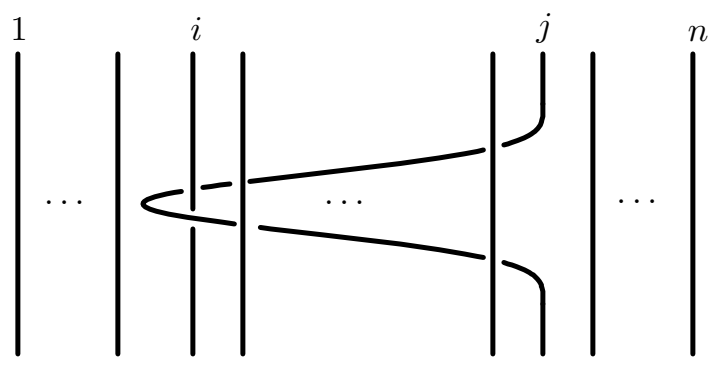

Figure 1. Pure braid $A_{i, j}$

braid $\beta$. If we set $g=g_{1}$, then $g$ is the identity on the $U_{i}$ 's and $\gamma\left(g ;\left(x_{1}, \ldots, x_{n}\right)\right)=\beta$ for $x_{i} \in U_{i}$. Then by setting $U=U_{1} \cup \cdots \cup U_{n}$, we have

$$
\begin{aligned}
& \lim _{p \rightarrow \infty} \frac{1}{p} \hat{\Gamma}_{n}(\phi)\left(g^{p}\right) \\
= & \lim _{p \rightarrow \infty} \frac{1}{p}\left(\int_{x \in X_{n}(U)} \phi\left(\gamma\left(g^{p} ; x\right)\right) d x+\int_{x \in X_{n}\left(D^{2}\right) \backslash X_{n}(U)} \phi\left(\gamma\left(g^{p} ; x\right)\right) d x\right) \\
= & \int_{x \in X_{n}(U)} \phi(\gamma(g ; x)) d x+\lim _{p \rightarrow \infty} \frac{1}{p} \int_{x \in X_{n}\left(D^{2}\right) \backslash X_{n}(U)} \phi\left(\gamma\left(g^{p} ; x\right)\right) d x .
\end{aligned}
$$

If we denote the first term of the equation by $Y$ and set $a_{i}=\operatorname{area}\left(U_{i}\right)$ and $[n]=$ $\{1, \ldots, n\}$, then $Y$ is written as

$$
\int_{x \in X_{n}(U)} \phi(\gamma(g ; x)) d x=\sum_{F:[n] \rightarrow[n]}\left(\prod_{i=1}^{n} a_{F(i)}\right) x_{F},
$$

where $x_{F}=\phi(\gamma(g ; x))$ for $x$ in the case when each $x_{i}$ is in $U_{F(i)}$. The real numbers $x_{F}$ have the following properties:

(i) For two maps $F$ and $G:[n] \rightarrow[n]$, if $\# F^{-1}(i)=\# G^{-1}(i)$ for each $1 \leq i \leq$ $n$, then $x_{F}=x_{G}$.

(ii) If a map $F:[n] \rightarrow[n]$ is bijective, then $x_{F}$ is non-zero.

Property (i) follows from the invariance of $\phi$ under conjugation, and property (ii) follows because $\phi(\beta)$ is non-zero. Therefore, the coefficient of $a_{1} \ldots a_{n}$ in $Y$ is nonzero. Since the polynomial $Y$ is not identically 0 , we can choose the $a_{i}$ 's such that $Y$ is non-zero.

Note that if we replace the $a_{i}$ 's by bigger ones, by fixing the ratio of any two of them the term $Y$ stays non-zero. On the other hand, the value $\phi(\gamma(g ; x))$ is bounded because of the construction of $g$, and we thus have

$$
\lim _{p \rightarrow \infty} \frac{1}{p} \int_{x \notin X_{n}(U)} \phi\left(\gamma\left(g^{p} ; x\right)\right) d x \rightarrow 0 \quad\left(\text { as } \quad a_{1}+\cdots+a_{n} \rightarrow \operatorname{area}\left(D^{2}\right)\right) .
$$

This completes the proof. 
As we noted in Remark 2.1 the homomorphism $\hat{\Gamma}_{n}$ maps any homomorphism on $P_{n}\left(D^{2}\right)$ to a homomorphism on $\operatorname{Diff}_{\Omega}^{\infty}\left(D^{2}, \partial D^{2}\right)$. Hence the homomorphism

$$
Q\left(P_{n}\left(D^{2}\right)\right) / H^{1}\left(P_{n}\left(D^{2}\right) ; \mathbb{R}\right) \rightarrow Q\left(\operatorname{Diff}_{\Omega}^{\infty}\left(D^{2}, \partial D^{2}\right)\right) / H^{1}\left(\operatorname{Diff}_{\Omega}^{\infty}\left(D^{2}, \partial D^{2}\right) ; \mathbb{R}\right)
$$

is also induced. By an argument similar to the proof of Theorem 1.2 the following proposition holds.

Proposition 2.2. The map

$$
Q\left(B_{n}\left(D^{2}\right)\right) / H^{1}\left(B_{n}\left(D^{2}\right) ; \mathbb{R}\right) \rightarrow Q\left(\operatorname{Diff}_{\Omega}^{\infty}\left(D^{2}, \partial D^{2}\right)\right) / H^{1}\left(\operatorname{Diff}_{\Omega}^{\infty}\left(D^{2}, \partial D^{2}\right) ; \mathbb{R}\right)
$$

induced by the composition $\Gamma_{n} \circ Q(i): Q\left(B_{n}\left(D^{2}\right)\right) \rightarrow Q\left(\operatorname{Diff}_{\Omega}^{\infty}\left(D^{2}, \partial D^{2}\right)\right)$ is injective.

The homomorphism $\Gamma_{n}: Q\left(P_{n}\left(D^{2}\right)\right) \rightarrow Q\left(\operatorname{Diff}_{\Omega}^{\infty}\left(D^{2}, \partial D^{2}\right)\right)$ can also be defined for the 2-sphere $S^{2}$ instead of $D^{2}$ as Gambaudo and Ghys mentioned in their paper. Let $\operatorname{Diff}_{\Omega}^{\infty}\left(S^{2}\right)_{0}$ be the identity component of the group of area-preserving diffeomorphisms of $S^{2}$. Then we can choose a pure braid $\gamma(g ; x) \in P_{n}\left(S^{2}\right)$ for any $g \in \operatorname{Diff}_{\Omega}^{\infty}\left(S^{2}\right)_{0}$ and for almost every $x \in X_{n}\left(S^{2}\right)$ as in the case of the 2-disk. Since the group Diff $\Omega_{\Omega}^{\infty}\left(S^{2}\right)_{0}$ is homotopy equivalent to $S O(3)$ [7, [8] and its fundamental group has order 2 , for any element $g$ of $\operatorname{Diff}_{\Omega}^{\infty}\left(S^{2}\right)_{0}$ there exist two homotopy classes of paths connecting the identity and $g$ in $\operatorname{Diff}_{\Omega}^{\infty}\left(S^{2}\right)_{0}$. However, for any homogeneous quasi-morphism $\phi$ on $P_{n}\left(S^{2}\right)$, the value $\phi(\gamma(g ; x))$ is independent of the choice of the path. In fact, the braid obtained from a path which represents the generator of $\pi_{1}\left(\operatorname{Diff}_{\Omega}^{\infty}\left(S^{2}\right)_{0}\right)$ has order 2 and is in the center of $P_{n}\left(S^{2}\right)$. Hence the homomorphism $\Gamma_{n}: Q\left(P_{n}\left(S^{2}\right)\right) \rightarrow Q\left(\operatorname{Diff}_{\Omega}^{\infty}\left(S^{2}\right)_{0}\right)$ is defined. Since the braid group $B_{n}\left(S^{2}\right)$ of the 2 -sphere on $n$-strands can be considered as a quotient group of the braid group $B_{n}\left(D^{2}\right)$, by an argument similar to the proof of Theorem 1.2 , we obtain the following theorem.

Theorem 2.3. The composition

$$
\Gamma_{n} \circ Q(i): Q\left(B_{n}\left(S^{2}\right)\right) \rightarrow Q\left(\operatorname{Diff}_{\Omega}^{\infty}\left(S^{2}\right)_{0}\right)
$$

is injective.

The homomorphism $Q(i)$ in the statement of Theorem 2.3 is the one induced from the inclusion $i: P_{n}\left(S^{2}\right) \rightarrow B_{n}\left(S^{2}\right)$.

\section{KERNEL OF THE HOMOMORPHism $\Gamma_{n}$}

The homomorphism $\Gamma_{n}: Q\left(P_{n}\left(D^{2}\right)\right) \rightarrow Q\left(\operatorname{Diff}_{\Omega}^{\infty}\left(D^{2}, \partial D^{2}\right)\right)$ itself is not injective although Theorem 1.2 holds. In this section we study the kernel of the homomorphism $\Gamma_{n}$.

Let $G$ be a group and $H$ its finite index subgroup. We denote by $\bar{\beta}$ the image of an element $\beta \in G$ by the natural projection $G \rightarrow G / H$. For each left coset $\sigma \in G / H$ of $G$ modulo $H$, we fix an element $\gamma_{\sigma} \in G$ such that $\overline{\gamma_{\sigma}}=\sigma$ and for any $\phi \in \hat{Q}(H)$ define the function $\hat{\mathcal{T}}(\phi): G \rightarrow \mathbb{R}$ by

$$
\hat{\mathcal{T}}(\phi)(\beta)=\frac{1}{(G: H)} \sum_{\sigma \in G / H} \phi\left(\gamma_{\overline{\beta \gamma_{\sigma}}}{ }^{-1} \beta \gamma_{\sigma}\right) .
$$

Since ${\gamma_{\overline{\beta \gamma_{\sigma}}}}^{-1} \beta \gamma_{\sigma}$ is in $H$, the function $\hat{\mathcal{T}}(\phi)$ is well-defined on $G$. 
Lemma 3.1. For any quasi-morphism $\phi$ on $H$, the function $\hat{\mathcal{T}}(\phi): G \rightarrow \mathbb{R}$ is also a quasi-morphism.

Proof. Since the equality

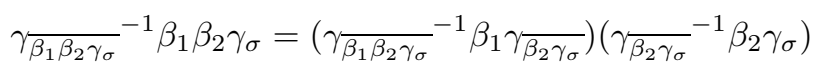

holds, we have the inequality

$$
\begin{aligned}
& \left|\hat{\mathcal{T}}(\phi)\left(\beta_{1} \beta_{2}\right)-\hat{\mathcal{T}}(\phi)\left(\beta_{1}\right)-\hat{\mathcal{T}}(\phi)\left(\beta_{2}\right)\right|
\end{aligned}
$$

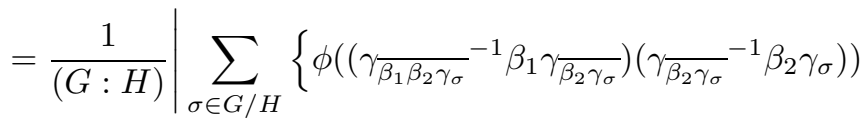

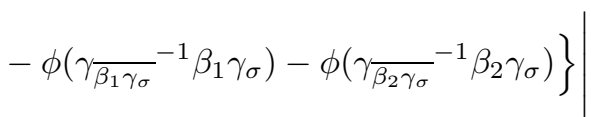

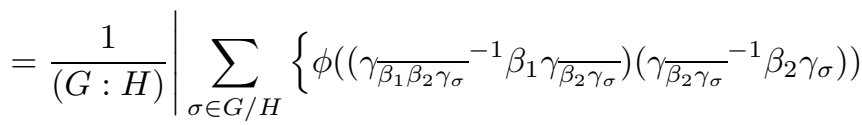

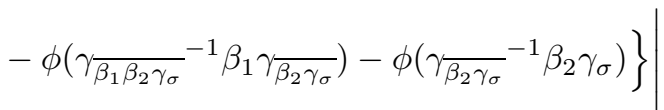

$$
\begin{aligned}
& \leq D(\phi) .
\end{aligned}
$$

Hence the function $\hat{\mathcal{T}}(\phi): G \rightarrow \mathbb{R}$ is also a quasi-morphism.

The map $\hat{\mathcal{T}}: \hat{Q}(H) \rightarrow \hat{Q}(G)$ is clearly $\mathbb{R}$-linear and induces a homomorphism $\mathcal{T}: Q\left(P_{n}\left(D^{2}\right)\right) \rightarrow Q\left(B_{n}\left(D^{2}\right)\right)$. Furthermore, the following proposition holds.

Proposition 3.2. The homomorphism $\mathcal{T}: Q(H) \rightarrow Q(G)$ is independent of the choice of $\gamma_{\sigma}$ 's.

Proof. Suppose that $\phi$ is a homogeneous quasi-morphism on $H$. If an element $\beta$ is in $H$, then $\overline{\gamma_{\sigma} \beta}=\sigma$ for each $\sigma \in G / H$. For any $\beta \in G$ there exists an integer $k$ such that $\beta^{k}$ is in $H$ and we have

$$
\begin{aligned}
\lim _{p \rightarrow \infty} \frac{1}{p} \hat{\mathcal{T}}(\phi)\left(\beta^{p}\right) & =\lim _{p^{\prime} \rightarrow \infty} \frac{1}{k p^{\prime}} \hat{\mathcal{T}}(\phi)\left(\beta^{k p^{\prime}}\right) \\
& =\lim _{p^{\prime} \rightarrow \infty} \frac{1}{(G: H) k p^{\prime}} \sum_{\sigma \in G / H} \phi\left(\gamma_{\sigma}^{-1} \beta^{k} \gamma_{\sigma}\right)^{p^{\prime}} \\
& =\frac{1}{(G: H) k} \sum_{\sigma \in G / H} \phi\left(\gamma_{\sigma}^{-1} \beta^{k} \gamma_{\sigma}\right) .
\end{aligned}
$$

Since $\phi$ is invariant under conjugations in $H$, the value $\phi\left(\gamma_{\sigma}^{-1} \beta^{k} \gamma_{\sigma}\right)$ depends only on $\sigma$.

Let $Q(i): Q(G) \rightarrow Q(H)$ be the homomorphism induced by the inclusion $i: H \rightarrow$ $G$. As a corollary to equality (3.1), we have the following.

Corollary 3.3. The composition $\mathcal{T} \circ Q(i): Q(G) \rightarrow Q(G)$ is the identity on $Q(G)$. Furthermore, we have the decomposition

$$
Q(H)=\operatorname{Ker}(\mathcal{T}) \oplus \operatorname{Im}(Q(i))
$$

as vector spaces. 
Remark 3.4. Of course, the homomorphism $\hat{\mathcal{T}}(\phi): G \rightarrow \mathbb{R}$ can be defined using the right coset $H \backslash G$ instead of $G / H$ by

$$
\hat{\mathcal{T}}(\phi)(\beta)=\frac{1}{(G: H)} \sum_{\sigma \in G / H} \phi\left(\gamma_{\sigma} \beta \gamma_{\bar{\gamma}_{\sigma} \beta}-1\right) .
$$

By an argument similar to the proof of Lemma 3.1 and Proposition 3.2, it is verified that this alternative definition is also well-defined and induces the same homomorphism $\mathcal{T}: Q(H) \rightarrow Q(G)$.

Remark 3.5. The homomorphism $\mathcal{T}: Q(H) \rightarrow Q(G)$ is just a straightforward generalization of the transfer map, and it is also introduced in [6] and [9].

Since the pure braid groups $P_{n}\left(D^{2}\right)$ and $P_{n}\left(S^{2}\right)$ are finite index subgroups of the braid groups $B_{n}\left(D^{2}\right)$ and $B_{n}\left(S^{2}\right)$, respectively, the homomorphisms

$$
\mathcal{T}: Q\left(P_{n}\left(D^{2}\right)\right) \rightarrow Q\left(B_{n}\left(D^{2}\right)\right) \quad \text { and } \quad \mathcal{T}: Q\left(P_{n}\left(S^{2}\right)\right) \rightarrow Q\left(B_{n}\left(S^{2}\right)\right)
$$

can be defined and Corollary 3.3 is true for $G=B_{n}\left(D^{2}\right), H=P_{n}\left(D^{2}\right)$ and $G=$ $B_{n}\left(S^{2}\right), H=P_{n}\left(S^{2}\right)$, respectively.

The following proposition is the main result of this section.

Proposition 3.6. The composition

$$
\Gamma_{n} \circ Q(i) \circ \mathcal{T}: Q\left(P_{n}\left(D^{2}\right)\right) \rightarrow Q\left(\operatorname{Diff}_{\Omega}^{\infty}\left(D^{2}, \partial D^{2}\right)\right)
$$

coincides with $\Gamma_{n}$. In particular, $\operatorname{Ker}\left(\Gamma_{n}\right)=\operatorname{Ker}(\mathcal{T})$ and $\operatorname{Im}\left(\Gamma_{n}\right)=\operatorname{Im}\left(\Gamma_{n} \circ Q(i)\right)$.

Proof. Let $\mathfrak{S}_{n}$ be the symmetric group of $n$ symbols. By equality (3.1), for any homogeneous quasi-morphism $\phi \in Q\left(P_{n}\left(D^{2}\right)\right)$ and any area-preserving diffeomorphism $g \in \operatorname{Diff}_{\Omega}^{\infty}\left(D^{2}, \partial D^{2}\right)$,

$$
\lim _{p \rightarrow \infty} \frac{1}{p} \hat{\Gamma}_{n} \circ Q(i) \circ \hat{\mathcal{T}}(\phi)\left(g^{p}\right)=\frac{1}{n !} \sum_{\sigma \in \mathfrak{S}_{n}} \lim _{p \rightarrow \infty} \frac{1}{p} \int_{x \in X_{n}\left(D^{2}\right)} \phi\left(\gamma_{\sigma} \gamma\left(g^{p} ; x\right) \gamma_{\sigma}^{-1}\right) d x .
$$

For any $\sigma \in \mathfrak{S}_{n}$ and almost all $x \in D^{2}$, we set the path $l:[0,1] \rightarrow X_{n}\left(D^{2}\right)$ by

$$
l(t)= \begin{cases}\left\{(1-2 t) x_{i}^{0}+2 t x_{i}\right\} & \left(0 \leq t \leq \frac{1}{2}\right), \\ \left\{(2-2 t) x_{i}+(2 t-1) x_{\sigma(i)}^{0}\right\} & \left(\frac{1}{2} \leq t \leq 1\right) .\end{cases}
$$

Considering the path $l$ as a loop in the quotient space $X_{n}\left(D^{2}\right) / \mathfrak{S}_{n}$, we define the braid $\beta(\sigma ; x)$ to be the braid represented by the loop $l$. Then by definition,

$$
\beta(\sigma ; x) \gamma\left(g ; \sigma^{-1}(x)\right) \beta\left(\sigma ; g_{*} x\right)^{-1}=\gamma(g ; x),
$$

where the symmetric group $\mathfrak{S}_{n}$ acts on $X_{n}\left(D^{2}\right)$ by the permutation

$$
\sigma\left(x_{1}, \ldots, x_{n}\right)=\left(x_{\sigma(1)}, \ldots, x_{\sigma(n)}\right) .
$$

Since the homomorphism $\mathcal{T}: Q\left(P_{n}\left(D^{2}\right)\right) \rightarrow Q\left(B_{n}\left(D^{2}\right)\right)$ is defined independently of the choice of braids $\gamma_{\sigma}$ 's, we may choose $\gamma_{\sigma}$ to be $\beta(\sigma ; x)$. Hence we have

$$
\begin{aligned}
\gamma_{\sigma} \gamma\left(g ; \sigma^{-1}(x)\right) \gamma_{\sigma}^{-1} & =\beta(\sigma ; x) \gamma\left(g ; \sigma^{-1}(x)\right) \beta(\sigma ; x)^{-1} \\
& =\gamma(g ; x) \beta\left(\sigma ; g_{*}(x)\right) \beta(\sigma ; x)^{-1} .
\end{aligned}
$$


Since the function $\phi(\beta(\sigma ; \cdot)): D^{2} \rightarrow \mathbb{R}$ is bounded on $D^{2}$, we have

$$
\begin{aligned}
& \lim _{p \rightarrow \infty} \frac{1}{p} \int_{x \in X_{n}\left(D^{2}\right)} \phi\left(\gamma_{\sigma} \gamma\left(g^{p} ; x\right) \gamma_{\sigma}^{-1}\right) d x \\
= & \lim _{p \rightarrow \infty} \frac{1}{p} \int_{x \in X_{n}\left(D^{2}\right)} \phi\left(\gamma_{\sigma} \gamma\left(g^{p} ; \sigma^{-1}(x)\right) \gamma_{\sigma}^{-1}\right) d x \\
= & \lim _{p \rightarrow \infty} \frac{1}{p} \int_{x \in X_{n}\left(D^{2}\right)} \phi\left(\gamma\left(g^{p} ; x\right)\right) d x .
\end{aligned}
$$

Therefore, by equality (3.2),

$$
\begin{aligned}
\lim _{p \rightarrow \infty} \frac{1}{p} \hat{\Gamma}_{n} \circ Q(i) \circ \hat{\mathcal{T}}(\phi)\left(g^{p}\right) & =\frac{1}{n !} \sum_{\sigma \in \mathfrak{S}_{n}} \lim _{p \rightarrow \infty} \frac{1}{p} \int_{x \in X_{n}\left(D^{2}\right)} \phi\left(\gamma\left(g^{p} ; x\right)\right) d x \\
& =\lim _{p \rightarrow \infty} \frac{1}{p} \hat{\Gamma}_{n}(\phi)\left(g^{p}\right),
\end{aligned}
$$

and thus we have $\Gamma_{n} \circ Q(i) \circ \mathcal{T}=\Gamma_{n}$.

Then obviously $\operatorname{Ker}(\mathcal{T}) \subseteq \operatorname{Ker}\left(\Gamma_{n}\right)$ and $\operatorname{Im}\left(\Gamma_{n}\right)=\operatorname{Im}\left(\Gamma_{n} \circ Q(i)\right)$ hold. If $\phi \in$ $\operatorname{Ker}\left(\Gamma_{n}\right)$, then

$$
\Gamma_{n} \circ Q(i) \circ \mathcal{T}(\phi)=\Gamma_{n}(\phi)=0,
$$

and hence $\mathcal{T}(\phi)=0$ by Theorem 1.2 . Thus we have $\operatorname{Ker}\left(\Gamma_{n}\right) \subseteq \operatorname{Ker}(\mathcal{T})$.

Remark 3.7. Proposition 3.6 also holds for $P_{n}\left(S^{2}\right)$ and $\operatorname{Diff}_{\Omega}^{\infty}\left(S^{2}\right)_{0}$ instead of $P_{n}\left(D^{2}\right)$ and $\operatorname{Diff}_{\Omega}^{\infty}\left(D^{2}, \partial D^{2}\right)$, respectively.

\section{ACKNOWLEDGMENTS}

This work is the main part of the author's doctoral thesis at the University of Tokyo, under the supervision of Professor Takashi Tsuboi. The author wishes to thank him for much helpful advice. The author also thanks Professors Étienne Ghys and Shigeyuki Morita for their warm encouragement and Professor Shigenori Matsumoto for valuable suggestions. The author is very grateful to the referee for a careful reading and for pointing out errors in the manuscript. The author was supported by JSPS Research Fellowships for Young Scientists (23.1352).

\section{REFERENCES}

[1] Michael Brandenbursky, On quasi-morphisms from knot and braid invariants, J. Knot Theory Ramifications 20 (2011), no. 10, 1397-1417, DOI 10.1142/S0218216511009212. MR2851716 (2012i:57002)

[2] Michael Brandenbursky and Jarek Kȩdra, On the autonomous metric on the group of areapreserving diffeomorphisms on the 2-disc, Algebr. Geom. Topol. 13 (2013), no. 2, 795-816. DOI 10.2140/agt.2913.13.795. MR3044593

[3] Michael Entov and Leonid Polterovich, Calabi quasimorphism and quantum homology, Int. Math. Res. Not. 30 (2003), 1635-1676, DOI 10.1155/S1073792803210011. MR.1979584 (2004e:53131)

[4] Jean-Marc Gambaudo and Étienne Ghys, Enlacements asymptotiques (French), Topology 36 (1997), no. 6, 1355-1379, DOI 10.1016/S0040-9383(97)00001-3. MR1452855 (98f:57050)

[5] Jean-Marc Gambaudo and Étienne Ghys, Commutators and diffeomorphisms of surfaces, Ergodic Theory Dynam. Systems 24 (2004), no. 5, 1591-1617, DOI 10.1017/S0143385703000737. MR2104597(2006d:37071) 
[6] A. V. Malyutin, Operators in the spaces of pseudocharacters of braid groups, Algebra i Analiz 21 (2009), no. 2, 136-165 (Russian, with Russian summary), DOI 10.1090/S1061-0022-1001094-0 English transl., St. Petersburg Math. J. 21 (2010), no. 2, 261-280. MR2553045 (2011b:20106)

[7] Jürgen Moser, On the volume elements on a manifold, Trans. Amer. Math. Soc. 120 (1965), 286-294. MR.0182927 (32 \#409)

[8] Stephen Smale, Diffeomorphisms of the 2-sphere, Proc. Amer. Math. Soc. 10 (1959), 621-626. MR0112149 (22 \#3004)

[9] Alden Walker, Surface maps into free groups, Thesis (Ph.D.)-California Institute of Technology, 2012, ProQuest LLC, Ann Arbor, MI. MR3034690

Graduate School of Mathematical Sciences, University of Tokyo, 3-8-1 Komaba, MEguro-Ku, TOKYO 153-8914, JAPAN

E-mail address: ishidat@ms.u-tokyo.ac.jp

Current address: Department of Mathematics, Kyoto University, Kitashirakawa Oiwake-cho, Sakyo-ku, Kyoto 606-8502, Japan

E-mail address: ishidat@math.kyoto-u.ac.jp 